\title{
The potential energy surface of $\left(\mathrm{NH}_{3}\right)_{2}$
}

\author{
Zdzislaw Latajka ${ }^{a)}$ and Steve Scheiner ${ }^{(b), c)}$ \\ Department of Chemistry and Biochemistry, Southern Illinois University, Carbondale, Ilinois 62901
}

\begin{abstract}
$A b$ initio calculations at the SCF and correlated levels are carried out to characterize the potential energy surface of the $\mathrm{NH}_{3}$ dimer. The two basis sets used are 4-31G* and a larger one containing two sets of $d$-functions on $N$ centers, 6-31G** (1p, 2d). The only minimum occurring on the surface is a cyclic $C_{2 h}$ structure in which the two $\mathrm{H}$-bonding protons are displaced $42^{\circ}$ from the $\mathrm{N}$ $-\mathrm{N}$ axis. The surface contains a very shallow valley along the direction leading from this geometry to a single linear $\mathrm{H}$ bond although there is no minimum corresponding to this arrangement. Despite the symmetrically nonpolar character of the cyclic geometry, the shallowness of the potential in the direction of the linear structure may allow zero point vibration effects to displace the minimum, thereby leading to the observed small dipole moment and its sensitivity to isotopic substitution.
\end{abstract}

\section{INTRODUCTION}

The structures of a number of simple hydrogen bonded systems have been well characterized both experimentally and theoretically. For example, molecular beam electric resonance spectroscopic (MBERS) data ${ }^{1}$ and $a b$ initio molecular orbital calculations ${ }^{2}$ agree that the equilibrium geometries of the $\mathrm{H}_{2} \mathrm{O}$ and $\mathrm{HF}$ dimers both contain a very nearly linear $\mathrm{H}$ bond. Presumably for this reason, it has been generally assumed that a similar arrangement is present in the dimer of $\mathrm{NH}_{3}$ as well. However, this assumption has not been verified experimentally as the structure of this complex has never been elucidated in the gas phase. Nor have theoretical methods been used to determine the true equilibrium geometry of $\left(\mathrm{NH}_{3}\right)_{2}$; previous ab initio calculations ${ }^{3-7}$ have for the most part simply assumed a linear $\mathrm{H}$ bond without sufficient testing.

Very recent MBERS data from Klemperer's group ${ }^{8}$ have called the above assumption into question. Since the dipole moment of the isolated $\mathrm{NH}_{3}$ monomer is $1.47 \mathrm{D},{ }^{9}$ one would expect a significantly larger value in the dimer if it contained a linear $\mathrm{H}$ bond due to a partial spatial alignment of the moments of the two subunits as well as the charge transfer which normally occurs in such bonds. However, the dipole moment determined by Klemperer $e t$ al. for the dimer is only $0.75 \mathrm{D}$, smaller even than that of the isolated monomer, thus ruling out the linear structure. ${ }^{8}$ Other alternatives that one might envisage include a cyclic configuration and a "trifurcated" geometry in which the three $\mathrm{H}$ atoms of one subunit are symmetrically disposed toward the $\mathrm{N}$ atom of the second. However, the dipole moment of the former is identically zero due to symmetry while that of the latter would be much larger than even the linear geometry since the dipole moments of the two subunits are collinear.

In order to shed some light on this issue, we have investigated an extended region of the potential energy surface (PES) of the $\mathrm{NH}_{3}$ dimer by $a b$ initio molecular orbital methods. Large polarized basis sets have been used in conjunction with inclusion of electron correlation effects and corrections for basis set superposition errors to avoid artifacts that might otherwise be encountered in the calculations. As shown be-

a) On leave from Institute of Chemistry, University of Wroclaw, Poland.

b) Address reprint requests to this author.

c) Recipient of NIH Research Career Development Award (1982-87). low, the lowest energy structure of $\left(\mathrm{NH}_{3}\right)_{2}$ contains a cyclic type of $\mathrm{H}$ bond. However, the PES is extremely flat in the direction of a configuration containing a linear $\mathbf{H}$ bond. Hence, the decrease in zero point vibrational energy occurring along this path may displace the structure of lowest total energy $(E+Z P E)$ away from the cyclic geometry, accounting for the small dipole moment observed.

\section{DETAILS OF CALCULATIONS}

The potential energy hypersurface was evaluated as a function of four intermolecular parameters. $R$ is the distance between nitrogen centers while $\alpha_{1}$ and $\alpha_{2}$ represent the angles between the $\mathrm{N}-\mathrm{N}$ internuclear axis and the local $C_{3}$ symmetry axis of each $\mathbf{N H}_{3}$ subunit, as illustrated in Fig. 1 . The last degree of freedom considered is a torsional rotation

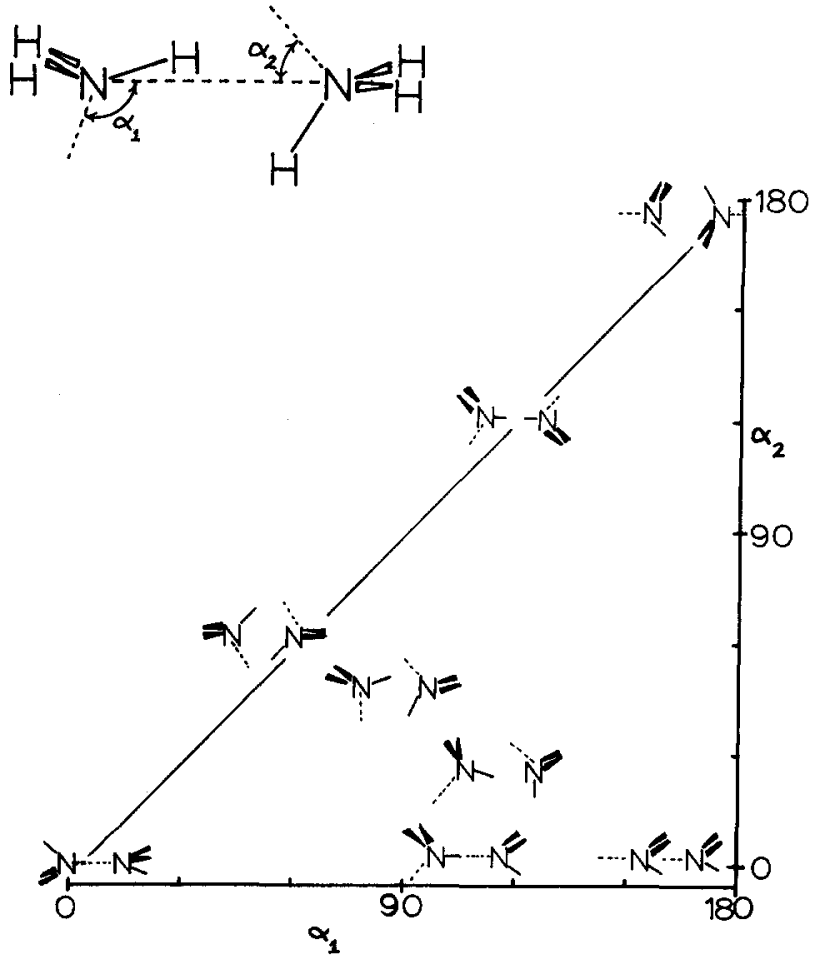

FIG. 1. Schematic diagram of various configurations of $\left(\mathrm{NH}_{3}\right)_{2}$ in terms of $\alpha_{1}$ and $\alpha_{2}$, defined above as the angles between the $\mathrm{N}-\mathrm{N}$ axis and the $C_{3}$ symmetry axes of the two $\mathrm{NH}_{3}$ subunits (denoted by the dotted lines). Geometries occurring above the diagonal are symmetrically related to those depicted in the lower right triangle. The linear configuration is of eclipsed type. 
of one $\mathrm{NH}_{3}$ molecule about its $C_{3}$ axis. The individual $\mathrm{NH}_{3}$ molecules were held fixed in their experimentally determined $^{10} \quad C_{3 v} \quad$ geometries: $r(\mathrm{NH})=1.0124 \AA$ and $\theta(\mathrm{HNH})=106.68^{\circ}$. We do not expect the latter assumption to introduce any significant errors into our surface due to the very weak nature of the interaction.

$A b$ initio calculations were carried out using the GAUSSIAN-80 computer $\operatorname{codes}^{11}$ with two different basis sets. 4$31 G^{*}$ was constructed by addition of a set of $d$-functions $(\zeta=0.8)$ to the $\mathrm{N}$ centers of the standard split-valence 4-31G set. ${ }^{12}$ The larger basis set, denoted $6-31 \mathrm{G}^{* *}(1 p, 2 d),{ }^{7}$ arises from the following modifications to $6-31 G^{* *}$. A second diffuse set of $d$ functions $(\xi=0.25)$ was added to the nitrogens while the exponent used for the single set of $p$ functions on $\mathbf{H}$ was 0.15 . This $6-31 G^{* *}(1 p, 2 d)$ set was used in our previous study of the $\mathrm{NH}_{3}$ dimer ${ }^{7}$ and yields excellent estimates of the electric multipole moments of the monomer, minimizes basis set superposition errors, and provides a suitable framework for inclusion of correlation effects. The dipole moments calculated for $\mathrm{NH}_{3}$ with these two basis sets are 1.92 and $1.50 \mathrm{D}$, respectively, as compared to the experimental value $^{9}$ of $1.47 \mathrm{D}$. Correlation was considered here via second-order Møller-Plesset perturbation theory (MP2) ${ }^{13}$ with frozen $1 s$ cores for the $\mathbf{N}$ atoms. The method of Boys and Bernardi ${ }^{14}$ was used to compute the basis set superposition error (BSSE).

\section{RESULTS}

In order to thoroughly investigate the potential energy surface in terms of three degrees of freedom, contour maps of the energy as a function of the intermolecular angles $\alpha_{1}$ and $\alpha_{2}$ were generated for each of a series of values of the distance between $\mathrm{N}$ centers, $\boldsymbol{R}$. The range of angles chosen for study varied between $0^{\circ}$ and $180^{\circ}$ and covered all chemically interesting conformations as well as all those which might be expected to be stable. Figure 1 displays our definitions of the $\alpha$ angles and illustrates some of the various geometries included in our calculations. The linear structure occurs at $\left(\alpha_{1}\right.$, $\left.\alpha_{2}\right)=\left(112^{\circ}, 0^{\circ}\right)$ and the trifurcated geometry at $\left(180^{\circ}, 0^{\circ}\right) . \mathrm{Cy}-$ clic arrangements lie along the diagonal, i.e., $\alpha_{1}=\alpha_{2}$; however, it is only when these two angles are in the range of perhaps $50^{\circ}-90^{\circ}$ that a stable structure might be expected. Since geometry $\left(\alpha_{2}, \alpha_{1}\right)$ is equivalent to $\left(\alpha_{1}, \alpha_{2}\right)$, structures above and to the left of the diagonal in Fig. 1 are duplicates of those shown and the contour surfaces in the following figures are thus symmetric with respect to this diagonal line. Note that the hydrogens of the two subunits are eclipsed relative to one another in the linear conformation. The effects on the PES of a torsional rotation required for a staggered linear configuration are investigated below.

Contour surfaces were generated using a grid of $10^{\circ}$ for the two angles. Due to the large number of points needed for these surfaces, the calculations were carried out at the SCF level using the smaller 4-31G* basis set. Figure 2 illustrates the contour surface determined for $R=3.20 \AA$, the shortest distance studied in detail. The lowest energy structure, labeled by a letter $C$, occurs along the diagonal at $\left(70^{\circ}, 70^{\circ}\right)$ and is of cyclic type, with $\mathrm{N}-\mathrm{-N}-\mathrm{H}$ angles equal to $42^{\circ}$. The linear geometry, represented by the $L$ label at $\left(112^{\circ}, 0^{\circ}\right)$ is $0.60 \mathrm{kcal} /$ mol higher in energy and is not associated with a minimum

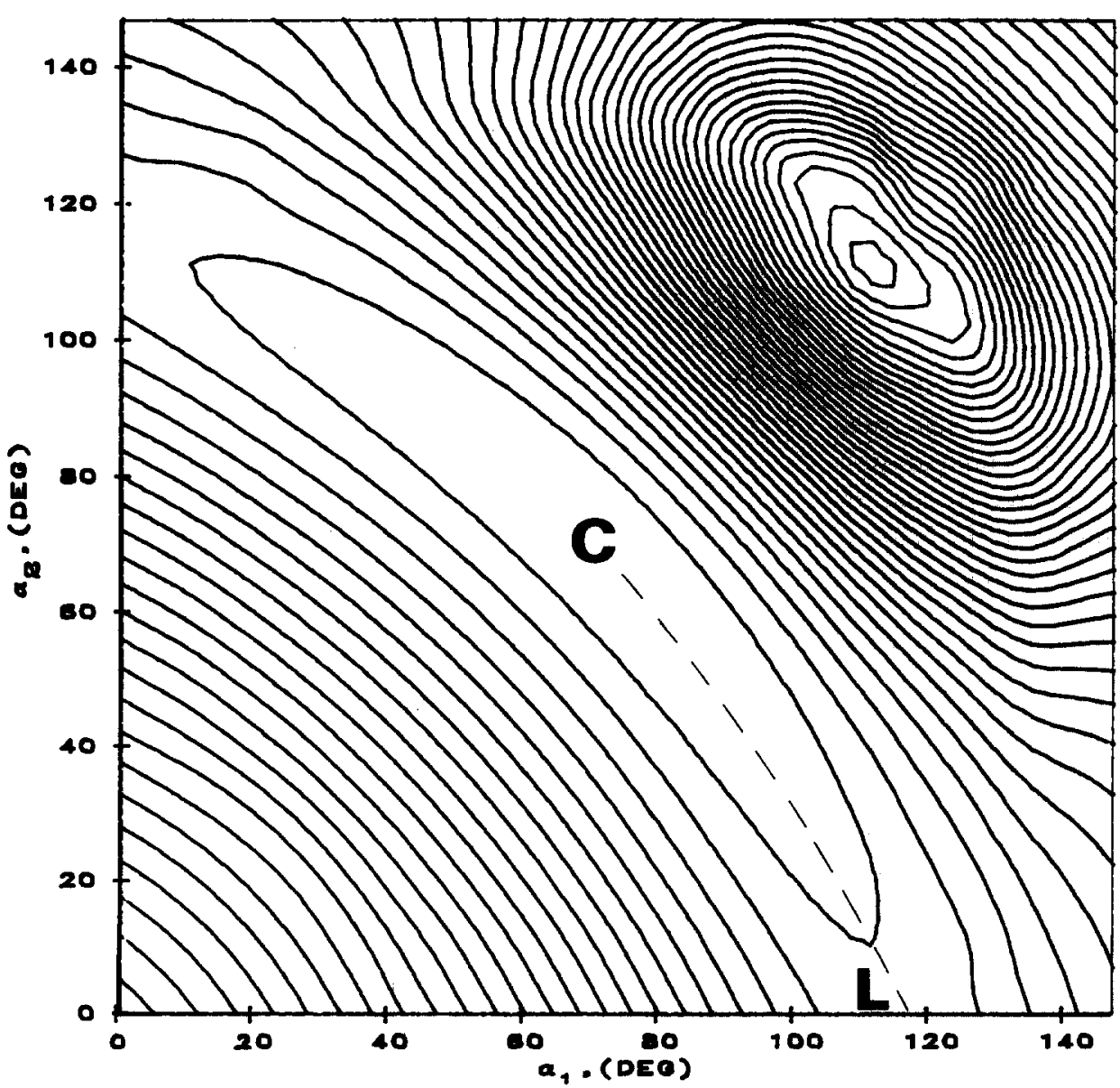

FIG. 2. Potential energy surface for $R=3.20 \AA$. Contour spacing is $0.5 \mathrm{kcal}$ mol. The broken line represents the lowest energy path emanating from the cyclic $(C)$ minimum, $3.43 \mathrm{kcal} / \mathrm{mol}$ lower in energy than a pair of isolated $\mathrm{NH}_{3}$ molecules. The subunits are in the eclipsed orientation. 
in the map. The broken line indicates the lowest energy path which converts the minimum energy cyclic geometry to a linear type of arrangement. Note that the path does not pass directly through the $L$ but slightly to its right instead. A maximum may be observed in the surface at approximately $\left(112^{\circ}, 112^{\circ}\right)$ due to the strong steric repulsion between the two $\mathrm{H}$ atoms (see Fig. 1). The $\left(0^{\circ}, 0^{\circ}\right)$ geometry is also rather high in energy as the dipole moments of the two $\mathrm{NH}_{3}$ subunits are antiparallel and their lone electron pairs point directly at one another with no intervening $\mathrm{H}$ atom. In fact, both of the latter two configurations are unbound, i.e., they lie substantially higher in energy than a pair of isolated $\mathrm{NH}_{3}$ molecules.

The contour surface calculated for $R=3.45 \AA$ is presented in Fig. 3 and contains a pair of symmetrically disposed minima, one of which occurs at $\left(105^{\circ}, 25^{\circ}\right)$. This structure is only marginally more stable $(0.1 \mathrm{kcal} / \mathrm{mol})$ than the cyclic geometry at $\left(70^{\circ}, 70^{\circ}\right)$. As in the previous case of $R=3.20 \AA$, there is no minimum corresponding precisely to the linear structure. Surfaces were generated also for values of $R$ intermediate between the two extremes of 3.20 and 3.45 $\AA$ at increments of $0.05 \AA$. For $R<3.35 \AA$, the surfaces resemble that in Fig. 2 with a single minimum at $\left(70^{\circ}, 70^{\circ}\right)$. For longer distances, two minima appear as in Fig. 3 but in no case are these minima lower in energy than the $C$ structure by more than $0.1 \mathrm{kcal} / \mathrm{mol}$. In summary, all the surfaces indicate that conversion between the cyclic and linear structures involves only very small changes in energy.

A more complete representation of the PES in all three degrees of freedom is displayed in Fig. 4 which is a composite of the surfaces for all values of $R$, viz. $R$ was optimized individually for each pair of angles $\left(\alpha_{1}, \alpha_{2}\right)$. In order to focus our attention on the lowest energy path, the $\alpha_{1}$ and $\alpha_{2}$ angles have been transformed into the symmetric and antisymmetric combinations labeling the axes. [The vertical axis of Fig. 4 corresponds to the segment of the diagonal from $\left(40^{\circ}, 40^{\circ}\right)$ to $\left(90^{\circ}, 90^{\circ}\right)$ of the previous figures.] Additional points have also been included which continue the path below the horizontal axes of Figs. 2 and 3 and thus beyond the linear structure. It should lastly be noted that the contours in Fig. 4 are spaced apart by $0.2 \mathrm{kcal} / \mathrm{mol}$ rather than the 0.5 increments used in the previous surfaces.

There is only one minimum in the full PES, corresponding to the cyclic structure. A long and very shallow valley extends away from this geometry and passes quite close to the linear arrangement, only $\sim 0.2 \mathrm{kcal} / \mathrm{mol}$ higher in energy. Even after following the dashed line path to the right edge of Fig. 4 , i.e., $\left(\alpha_{1}, \alpha_{2}\right) \cong\left(130^{\circ},-40^{\circ}\right)$, the energy has risen by only $1.0 \mathrm{kcal} / \mathrm{mol}$. It is therefore a central conclusion of these calculations that the $\mathrm{NH}_{3}$ dimer is extremely flexible with a low energy conversion path between the cyclic and linear structures.

It should be noted finally that the trifurcated structure, indicated by the $T$ in the upper right corner of Fig. 4, does not correspond to a minimum in the surface and lies 2.2 $\mathrm{kcal} / \mathrm{mol} \mathrm{higher} \mathrm{in} \mathrm{energy} \mathrm{than} \mathrm{the} \mathrm{cyclic} \mathrm{minimum.} \mathrm{In} \mathrm{addi-}$ tion, the optimized internitrogen distance in the trifurcated geometry is $0.5 \AA$ longer than in the equilibrium $C$ structure.

To ensure that the above conclusions are not an artifact

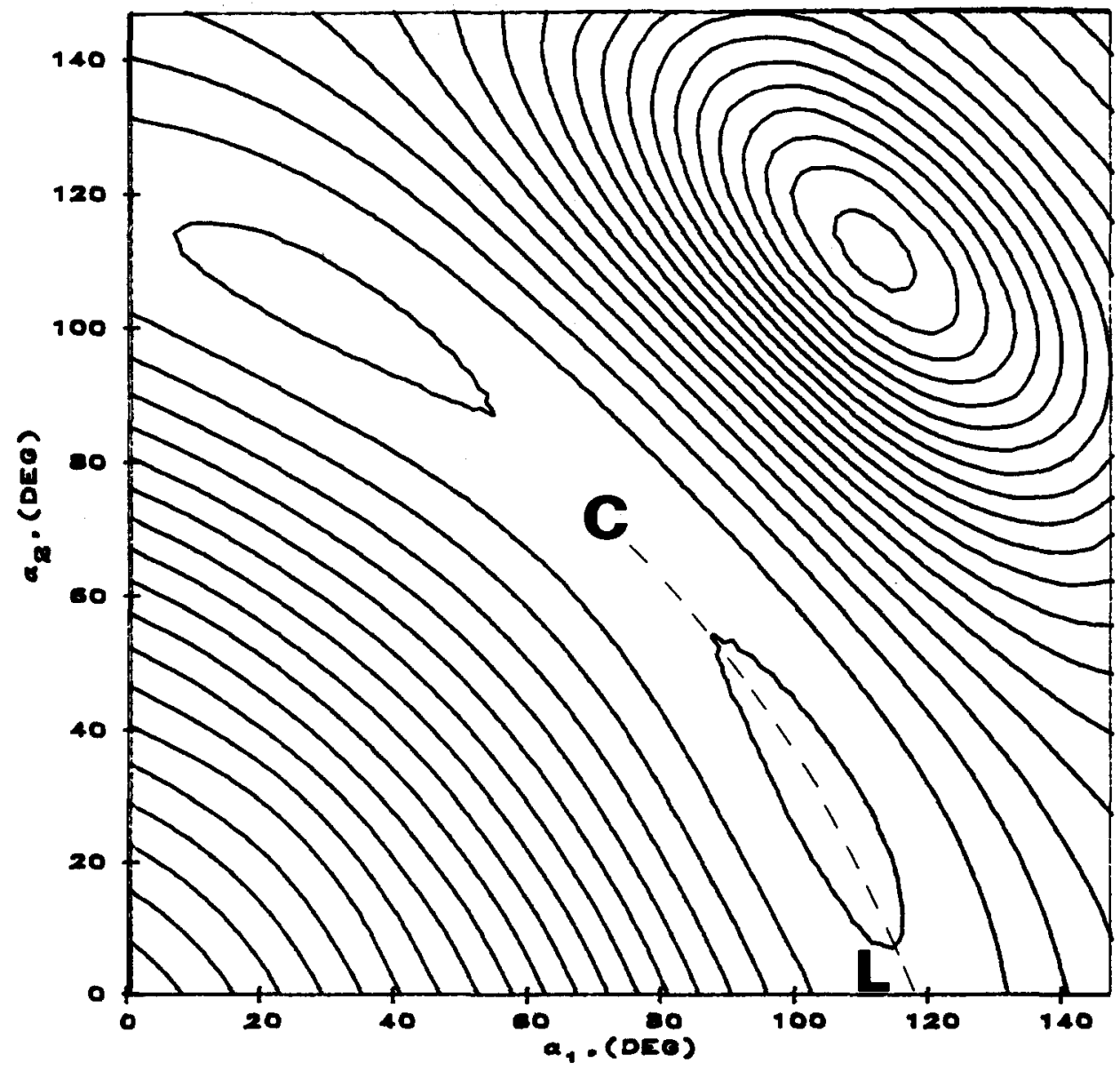

FIG. 3. Potential energy surface for $R=3.45 \AA$ with contour interval 0.5 $\mathrm{kcal} / \mathrm{mol}$. The two minima occur at $\left(\alpha_{1}\right.$, $\left.\alpha_{2}\right)=\left(105^{\circ}, 25^{\circ}\right)$ and $\left(25^{\circ}, 105^{\circ}\right)$. The energy of the $C$ structure is $-3.23 \mathrm{kcal} / \mathrm{mol}$. 


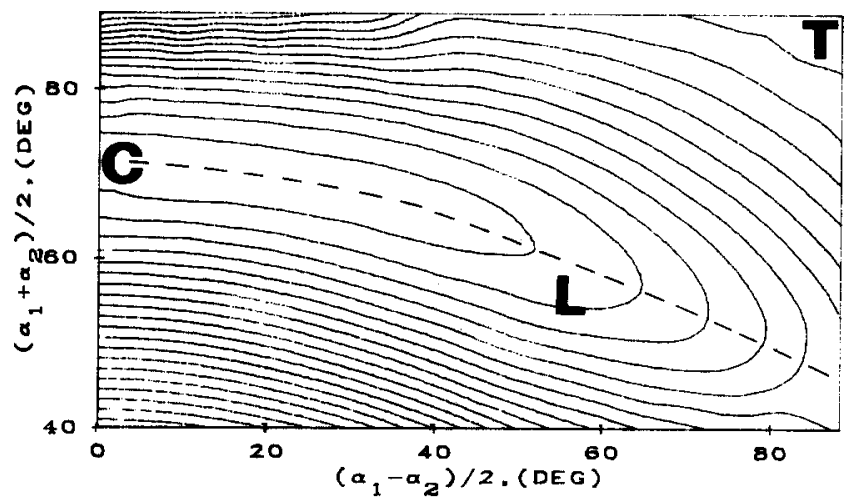

FIG. 4. PES including optimization of $R$ at each point $\left(\alpha_{1}, \alpha_{2}\right)$. The spacing between contours is $0.2 \mathrm{kcal} / \mathrm{mol}$. The horizontal and vertical axes have been rotated to display a longer stretch of the conversion path, indicated by the dashed line. The $T$ label in the upper right corner refers to the "trifurcated" geometry with $\left(\alpha_{1}, \alpha_{2}\right)=\left(180^{\circ}, 0^{\circ}\right)$. Molecules are in the eclipsed orientation.

of the basis set being used or failure to include effects of electron correlation, additional calculations were carried out along the conversion path using the 6-31G** $(1 p, 2 d)$ basis set coupled with the MP2 technique. The results are presented in Table I for both basis sets used here. The third column lists the intermolecular distances optimized at the SCF/4-31G* level along the conversion path. It is clear that the two subunits are closest to one another in the cyclic arrangement $\left(70^{\circ}, 70^{\circ}\right)$ and move progressively further apart along the path, stretching by $0.2 \AA$ in the linear structure. The same trend is evident at the MP2/6-31G** $(1 p, 2 d)$ level although the values of $R$ in the eighth column of Table I are uniformly smaller by $0.1 \AA$, due primarily to the inclusion of dispersion in the optimization.

With regard to the $4-31 \mathrm{G}^{*}$ basis set, the fourth column of Table $I$ indicates that the $C$ minimum is bound relative to the two isolated $\mathrm{NH}_{3}$ monomers by $3.44 \mathrm{kcal} / \mathrm{mol}$ at the SCF level. Correlation contributes another $1.40 \mathrm{kcal} / \mathrm{mol}$, leading to a total value of $4.84 \mathrm{kcal} / \mathrm{mol}$, as shown in the succeeding columns. The $C$ geometry is more stable than $L$ by $0.2 \mathrm{kcal} / \mathrm{mol}$ at the SCF level; an additional 0.2 is added to this difference by MP2 terms. With the larger 6-31G** $(1 p, 2 d)$ basis set, the greater stability of the $C$ structure is more clearly attributed to correlation. At the SCF level, $C$ and $L$ are nearly identical in energy ${ }^{15}$ whereas the difference in correlation energy is about $0.2 \mathrm{kcal} / \mathrm{mol}$.

An important distinction between the two basis sets is that the larger one ascribes half the total binding energy of the dimer to correlation while the same contribution calculated with the smaller set is only about $30 \%$. The large SCF/ 4-31 $\mathrm{G}^{*}$ binding energies are due in part to the overestimate of the dipole moments of the monomers with this basis set. The $6-31 \mathrm{G}^{* *}(1 p, 2 d)$ basis set provides a more realistic assessment of the SCF binding energy and also a better framework for measuring the contribution of correlation. ${ }^{7}$

Despite the differences noted above, calculations at both the SCF/4-31G* and MP2/6-31G** $(1 p, 2 d)$ levels agree that the cyclic structure is the only true minimum in the PES. However, the dimer is extremely flexible and may follow a very flat valley in configuration space and convert to a linear arrangement at a cost of only $0.2 \mathrm{kcal} / \mathrm{mol}$ with no intervening barrier. This lack of rigidity includes the intermolecular distance as well as angular characteristics. For example, stretches or contractions of $R$ by $0.05 \AA$ from its equilibrium value at any point along the conversion path involve energy increases of less than $0.03 \mathrm{kcal} / \mathrm{mol}$, corresponding to stretching force constants below $0.12 \mathrm{mdyn} / \AA$.

As a final check, the basis set superposition error (BSSE) was calculated ${ }^{14}$ with the $6-31 \mathrm{G}^{* *}(1 p, 2 d)$ basis set. At the SCF level, values obtained were 0.82 and $0.75 \mathrm{kcal} / \mathrm{mol}$ for the cyclic and linear geometries, respectively, whereas these quantities increased to 1.64 and 1.65 at the SCF + MP2 level. It is clear that BSSE does not preferentially stabilize one geometry over the other and therefore does not affect our conclusions concerning the small energy difference between the two configurations.

A final degree of freedom considered is the torsional rotation of the $\mathrm{NH}_{3}$ subunits about their respective $C_{3}$ axes. As pointed out above, the linear configuration in Fig. 1 contains an eclipsed arrangement of the hydrogens of the two molecules. A $60^{\circ}$ rotation of the right subunit would produce

TABLE I. Calculated interaction energies and dipole moments along conversion path.

\begin{tabular}{|c|c|c|c|c|c|c|c|c|c|c|c|c|}
\hline \multirow[b]{3}{*}{$\alpha_{1}$} & \multirow[b]{3}{*}{$\alpha_{2}$} & & \multicolumn{5}{|c|}{$4-31 G^{*}$} & \multicolumn{5}{|c|}{$6-31 \mathrm{G}^{* *}(1 p, 2 d)$} \\
\hline & & & \multirow[t]{2}{*}{$R,{ }^{\mathrm{a}}(\AA)$} & \multicolumn{3}{|c|}{$-\Delta E(\mathrm{kcal} / \mathrm{mol})$} & \multirow[t]{2}{*}{$\mu(\mathrm{D})$} & \multirow[t]{2}{*}{$R,{ }^{b}(\AA)$} & \multicolumn{3}{|c|}{$-\Delta E(\mathrm{kcal} / \mathrm{mol})$} & \multirow[t]{2}{*}{$\mu(\mathrm{D})$} \\
\hline & & & & SCF & MP2 & $\mathrm{SCF}+\mathrm{MP2}$ & & & SCF & MP2 & $\mathrm{SCF}+\mathrm{MP2}$ & \\
\hline 70 & 70 & $\mathrm{C}$ & 3.25 & 3.44 & 1.40 & 4.84 & 0.0 & 3.15 & 2.13 & 2.13 & 4.26 & 0.0 \\
\hline 81 & 60 & & 3.25 & 3.44 & 1.41 & 4.85 & 0.87 & & & & & \\
\hline 89 & 50 & & 3.32 & 3.42 & 1.29 & 4.71 & 1.59 & 3.21 & 2.13 & 2.03 & 4.16 & 1.33 \\
\hline 97 & 40 & & 3.35 & 3.41 & 1.28 & 4.69 & 2.21 & & & & & \\
\hline 103 & 30 & & 3.35 & 3.41 & 1.29 & 4.70 & 2.72 & 3.28 & 2.17 & 1.95 & 4.12 & 2.25 \\
\hline 108 & 20 & & 3.40 & 3.39 & 1.24 & 4.63 & 3.12 & & & & & \\
\hline 113 & 10 & & 3.45 & 3.33 & 1.18 & 4.51 & 3.43 & & & & & \\
\hline 112 & 0 & $\mathbf{L}$ & 3.45 & 3.21 & 1.18 & 4.39 & 3.58 & 3.34 & 2.16 & 1.89 & 4.05 & 2.94 \\
\hline 116 & 0 & & 3.45 & 3.24 & 1.18 & 4.42 & 3.66 & 3.35 & 2.12 & 1.83 & 3.95 & 2.96 \\
\hline 124 & -20 & & 3.48 & 2.92 & 1.13 & 4.05 & 3.99 & 3.35 & 1.82 & 1.81 & 3.63 & 3.25 \\
\hline 131 & -40 & & 3.50 & 2.45 & 1.04 & 3.49 & 4.05 & 3.38 & 1.49 & 1.72 & 3.21 & 3.26 \\
\hline
\end{tabular}

- Optimized at SCF/4-31G* level.

${ }^{b}$ Optimized at MP2/6-31G** $(1 p, 2 d)$ level. 
a staggered conformation which would presumably be somewhat different in energy. In order to check the effects of such a rotation, a "staggered" PES was generated just as in the previous eclipsed case except that the right subunit was rotated by $60^{\circ}$ around its $C_{3}$ axis relative to its orientation in Fig. 1. As before, $R$ was optimized for each pair of angles $\alpha_{1}$ and $\alpha_{2}$ chosen. The portion of the staggered surface containing the cyclic and linear geometries is presented in Fig. 5. Comparison with the analogous "eclipsed" PES in Fig. 4 illustrates that the $60^{\circ}$ rotation has displaced the minimum away from the cyclic geometry and towards the linear structure. The minimum in the staggered surface occurs at $\left(\alpha_{1}\right.$, $\left.\alpha_{2}\right)=\left(109^{\circ}, 18^{\circ}\right)$, intermediate between the cyclic and linear configurations. However, the latter minimum is slightly higher in energy $(0.08 \mathrm{kcal} / \mathrm{mol})$ than the cyclic eclipsed structure to which it would freely convert with no energy barrier if the specification of a staggered arrangement were removed.

An insight into the source of the discrepancy between the staggered and eclipsed surfaces is provided in Fig. 6 which is a contour representation of their difference. That is, for each pair of angles $\left(\alpha_{1}, \alpha_{2}\right)$, the energy of the eclipsed configuration with its optimized value of $R$ was subtracted from that of the staggered arrangement with its own $R$. Hence, Fig. 6 may be considered to illustrate the barrier to rotation of the right $\mathrm{NH}_{3}$ subunit about its local $C_{3}$ axis. The solid contours represent positive barriers, i.e., the staggered structure is higher in energy than the eclipsed. Note the high values in the vicinity of the cyclic structure. This large energy difference is not surprising in view of the fact that a $60^{\circ}$ rotation would disrupt half of the cyclic bond by turning one of the two $\mathrm{H}$-bonding protons away from the $\mathrm{N}$ atom to which it is bonded. On the other hand, the region in the vicinity of the linear structure is quite insensitive to conversion from eclipsed to staggered. In other words, there is free rotation of the right molecule about its $C_{3}$ axis. The broken contours in the upper and lower sections of Fig. 6 indicate greater stability for the staggered arrangement. However,

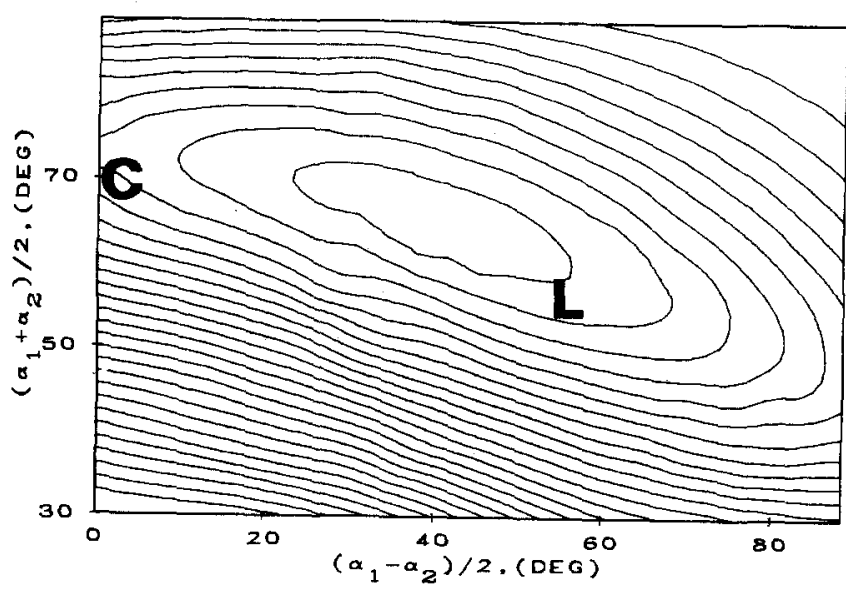

FIG. 5. PES of the "staggered" arrangement of the two $\mathrm{NH}_{3}$ subunits calculated at the SCF/4-31G* level. Conventions are identical to those in Fig. 4 with contour spacing $0.2 \mathrm{kcal} / \mathrm{mol}$. The minimum occurs at approximately $\left(\alpha_{1}, \alpha_{2}\right)=\left(109^{\circ}, 18^{\circ}\right)$ with $\Delta E=-3.36 \mathrm{kcal} / \mathrm{mol}$. The $C$ label does not correspond to a minimum on the staggered PES but is used merely to indicate the position of the cyclic minimum in the eclipsed surface.

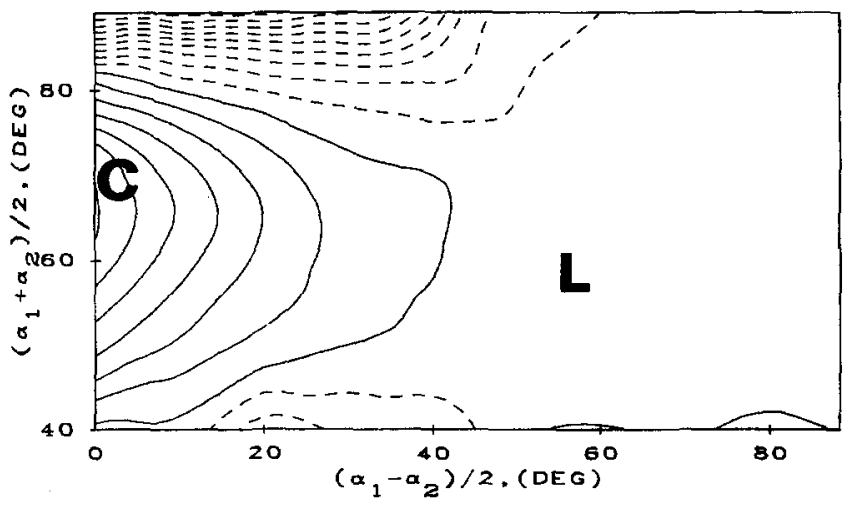

FIG. 6. $E$ (staggered) $-E$ (eclipsed) calculated as difference between Figs. 4 and 5 . Contour spacing is $0.1 \mathrm{kcal} / \mathrm{mol}$. Solid contours indicate higher energy for staggered conformation and broken contours for the eclipsed geometry.

these regions are of little interest due to their high energy and their distance from the path connecting the cyclic and linear geometries.

In summary, the conversion from the eclipsed to staggered arrangement destabilizes the cyclic configuration while leaving the energy of the linear structure essentially unchanged. The eclipsed cyclic geometry represents the only true minimum in the PES.

\section{DISCUSSION}

The shallow path leading away from the global minimum agrees quite well with Klemperer's observation ${ }^{8}$ of a nonrigid structure for $\left(\mathrm{NH}_{3}\right)_{2}$. Also consistent is the experimental finding that neither $\mathrm{NH}_{3}$ subunit inverts in the complex. ${ }^{8 b}$ Such an inversion would seriously perturb the $\mathbf{H}$ bonding pattern of geometries in the vicinity of the cyclic structure.

On the other hand, the nonzero dipole moment measured in the gas phase ${ }^{8,16}$ appears inconsistent with our finding of the cyclic structure, with its zero moment, as the global minimum. We believe this discrepancy may be reconciled by consideration of zero point vibrational energies. The stretching force constant decreases from its maximal value of $0.12 \mathrm{mdyn} / \AA$ for the cyclic geometry to 0.085 in the linear structure. With regard to angular components, it is clear from Fig. 4 that the energy rises most steeply with perpendicular deviations from the dashed line path in the vicinity of the $C$ structure. Lastly, the torsional barriers presented in Fig. 6 are greatest for $C$ as well. Since the zero point energy diminishes with decreases in the various force constants, motion along the low energy conversion pathway away from the minimum energy $C$ structure will lower the total ZPE. Since the energy $E$ rises so slowly along this path, the total of $E+$ ZPE may be displaced away from $C$ to some extent, leading to an equilibrium structure with $C_{s}$ rather than $C_{2 h}$ symmetry. The SCF dipole moments presented in Table I indicate that the experimentally observed $\mu$ of $0.75 \mathrm{D}$ would occur for a displacement of perhaps only $10-15^{\circ}$ from $\left(70^{\circ}, 70^{\circ}\right)$. This hypothesis is supported by the smaller dipole moment of $0.57 \mathrm{D}$ measured for $\left(\mathrm{ND}_{3}\right)_{2} \cdot{ }^{8}$ The higher mass of the deuterated system lowers the vibrational levels, reducing 
the ability of ZPE to shift the minimum of $E+Z P E$ away from the $C$ structure.

From their structural data, Klemperer et al. ${ }^{8(b)}$ hypothesized a structure for the dimer similar to our cyclic geometry except that one molecule is rotated by $60^{\circ}$ about its $C_{3}$ axis. On the other hand, these workers indicated that they could not determine the value of their torsional angles $\chi$ from their data; changing their guess of $\chi_{1}$ from $180^{\circ}$ to $120^{\circ}$ would bring their structure in closer coincidence with our minimum. However, their predicted structure contains angles $\left(\alpha_{1}, \alpha_{2}\right)=\left(65^{\circ}, 49^{\circ}\right)$, somewhat different than our cyclic structure. Rather than lying along the reaction path, this geometry occurs in a fairly high energy region of our PES, 1 $\mathrm{kcal} / \mathrm{mol}$ less stable than our $C$ minimum at the MP2/6$31 \mathrm{G}^{* *}(1 p, 2 d)$ level. Nor does it represent a local minimum as no energy barrier separates it from the dashed-line conversion path in Fig. 4.

The distance between the centers of mass measured by Klemperer et al. is $3.337 \AA$, $^{8(b)}$ significantly longer than the MP2/6-31G** $(1 p, 2 d)$ value of $3.20 \AA$ for the cyclic geometry (although somewhat shorter than the distance $3.39 \AA \AA$ in the linear structure). Part of this discrepancy arises from basis set truncation and associated superposition errors. Probably more important, however, is the fact that the theoretical values refer to equilibrium distances, in contrast to the vibrationally averaged experimental measurement. The very low force constant for stretching of the intermolecular distance mentioned above would be expected to yield a substantially smaller $R_{e}$ than $R_{0}$.

To our knowledge, only two sets of workers have previously carried out any $a b$ initio comparison of linear and cyclic geometries of the $\mathrm{NH}_{3}$ dimer. ${ }^{5}$ In contrast to our results, Jorgensen and Ibrahim ${ }^{5(\mathrm{a})}$ found the former structure to be more stable. However, their use of a minimal STO-3G basis set and failure to include any correlation effects cast doubt on their results, especially in light of their finding of a very short internitrogen distance $(3.067 \AA)$ in the linear structure. This close approach is indicative of a very large BSSE which provides a spurious addition to the true interaction energy. Indeed, our previous recalculation showed that over $80 \%$ of the binding energy of their linear complex is due to this artifact. ${ }^{7}$ It should also be mentioned that Jorgensen and Ibrahim did not attempt to study the PES of $\left(\mathrm{NH}_{3}\right)_{2}$ or to determine if their linear and cyclic structures were true minima or higher-order stationary points. Hirao et al..$^{s(b)}$ performed their calculations at a somewhat higher level of theory including full geometry optimizations and some consideration of correlation effects. Their data confirm our finding of a small energy difference between the cyclic and linear geometries. As a second point of agreement, the cyclic structure was found more stable by $0.1 \mathrm{kcal} / \mathrm{mol}$ with their most complete basis set, 6-31G**.

Our finding here that the cyclic conformation is a minimum in the PES of $\left(\mathrm{NH}_{3}\right)_{2}$ stands in marked contrast to previous observations of analogous systems such as $\left(\mathrm{H}_{2} \mathrm{O}\right)_{2}$ and $(\mathrm{HF})_{2}$ where the cyclic structure represents a saddle point. Moreover, the linear geometry is not a minimum for $\left(\mathrm{NH}_{3}\right)_{2}$ whereas the equilibrium structures of the two latter dimers both contain linear (or nearly so) $\mathbf{H}$ bonds. This dif- ference may arise partially from the electrostatic interaction between the dipole moments of the two subunits. The dipole-dipole term in the multipole expansion of the electrostatic energy may be expressed as a function of the orientations of the dipoles with respect to an intermolecular axis. ${ }^{17}$ In the case of $(\mathbf{H F})_{2}$, the dipoles are lined up quite favorably in the linear structure (despite a bend of the proton-accepting subunit) whereas the dipole-dipole term is approximately zero in the cyclic geometry. ${ }^{2(c)}$ Although the interaction between dipole moments in the cyclic water dimer is stabilizing, the alignment is substantially superior in the linear configuration..$^{2(c)}$ The situation is notably different in the case of $\left(\mathrm{NH}_{3}\right)_{2}$ where the dipole-dipole term for our cyclic dimer $\left(70^{\circ}, 70^{\circ}\right)$ is almost precisely equal to its value in the linear structure $\left(112^{\circ}, 0^{\circ}\right)$. Hence, whereas the electrostatic interaction between subunit dipole moments favors the linear geometry of $(\mathrm{HF})_{2}$ and $\left(\mathrm{H}_{2} \mathrm{O}\right)_{2}$, the cyclic and linear structures of $\left(\mathrm{NH}_{3}\right)_{2}$ are not clearly differentiated in this regard.

Of course the above picture is somewhat oversimplified. The smaller intermolecular distance in the cyclic structure would further enhance the dipole-dipole interaction energy as well as higher-order terms in the multipole expansion. The relative stabilities of two bent $\mathbf{H}$ bonds in the cyclic geometry vs a single nearly linear one would also be affected by associated differences in charge transfer, polarization, exchange, and dispersion energies. The fundamental source of the difference in stability between the linear and cyclic conformations of various $\mathrm{H}$-bonded dimers will be the subject of future investigations in this laboratory.

Note added in proof: Very recent calculations by Frisch et al. ${ }^{18}$ which considered terms up to fourth order in the MP expansion and zero-point vibrational effects, have confirmed the small energy difference between the cyclic and linear structures. The question of which was more stable was found to be sensitive to the presence of a diffuse $s p$ shell in the $\mathbf{N}$ basis set. One difference between Ref. 18 and the work described here is that Frisch et al. carried out optimizations only at the SCF level and thereby neglected the effects of correlation on the intermolecular distance.

\section{ACKNOWLEDGMENTS}

We are grateful to W. Klemperer for very helpful discussions and for sending us a copy of Ref. 8(b) prior to publication. Thanks are extended to E. A. Hillenbrand for assistance with the graphical presentation of data. Some of the calculations were performed on the SIU Theoretical Chemistry Computer, funded in part by a grant from the Harris Corporation. We thank John Yates for making available a Harris version of GAUSSIAN-80. This work was supported by grants from the National Institutes of Health (GM29391 and AM01059) and by the Research Corporation.

'(a) T. R. Dyke, B. J. Howard, and W. Klemperer, J. Chem. Phys. 56, 2442 (1972); (b) T. R. Dyke, ibid. 66, 492 (1977); T. R. Dyke, K. M. Mack, and J. S. Muenter, ibid. 66, 498 (1977); J. A. Odutola and T. R. Dyke, ibid. 72, $5062(1980)$. 
${ }^{2}$ (a) D. R. Yarkony, S. V. O'Neil, H. F. Schaefer, C. P. Baskin, and C. F. Bender, J. Chem. Phys. 60, 855 (1974); H. Lischka, Chem. Phys. Lett. 66, 108 (1979); J. F. Gaw, Y. Yamaguchi, M. A. Vincent, and H. F. Schaefer, J. Am. Chem. Soc. 106, 3133 (1984); D. W. Michael, C. E. Dykstra, and J. M. Lisy, J. Chem. Phys. 81, 5998 (1984); (b) H. Popkie, H. Kistenmacher, and E. Clementi, ibid. 59, 1325 (1973); H. Kistenmacher, G. C. Lie, H. Popkie, and E. Clementi, ibid. 61, 546 (1974); O. Matsuoka, E. Clementi, and M. Yoshimine, ibid. 64, 1351 (1976); (c) R. C. Kerns and L. C. Allen, J. Am. Chem. Soc. 100, 6587 (1978).

${ }^{3}$ P. A. Kollman and L. C. Allen, J. Am. Chem. Soc. 93, 4991 (1971); W. C. Topp and L. C. Allen, ibid. 96, 5291 (1974); P. Kollman, J. McKelvey, A. Johansson, and S. Rothenberg, ibid. 97,955 (1975); H. Umeyama and K. Morokuma, ibid. 99, 1316 (1977); K. Ohta, Y. Yoshioka, K. Morokuma, and K. Kitaura, Chem. Phys. Lett. 101, 12 (1983); N. C. Baird, Int. J. Quantum Chem. 1, 49 (1974).

${ }^{4}$ A. Hinchliffe, D. G. Bounds, M. L. Klein, I. R. McDonald, and R. Righini, J. Chem. Phys. 74, 1211 (1981).

s(a) W. L. Jorgensen and M. Ibrahim, J. Am. Chem. Soc. 102, 3309 (1980); (b) K. Hirao, T. Fujikawa, H. Konishi, and S. Yamabe, Chem. Phys. Lett. 104, 184 (1984).

'J. A. Pople, Faraday Discuss. Chem. Soc. 73, 7 (1982).

'Z. Latajka and S. Scheiner, J. Chem. Phys. 81, 407 (1984).

${ }^{8}$ (a)G. T. Fraser, D. D. Nelson, Jr., A. Charo, and W. Klemperer, J. Chem.
Phys. 82, 2535 (1985); (b) D. D. Nelson, Jr., G. T. Fraser, and W. Klemperer, ibid. (submitted).

${ }^{9}$ E. A. Cohen and A. L. Poynter, J. Mol. Spectrosc. 53, 131 (1974).

${ }^{10}$ W. S. Benedict and E. K. Plyler, Can. J. Chem. 35, 1235 (1957).

${ }^{11}$ J. S. Binkley, R. A. Whiteside, R. Krishnan, R. Seeger, D. J. DeFrees, H. B. Schlegel, L. R. Kahn, and J. A. Pople, QCPE 1981, 406.

${ }^{12}$ J. B. Collins, P. von R. Schleyer, J. S. Binkley, and J. A. Pople. J. Chem. Phys. 64, 5142 (1976); R. Ditchfield, W. J. Hehre, and J. A. Pople, ibid. 54, 724 (1971).

${ }^{13}$ J. S. Binkley and J. A. Pople, Int. J. Quantum Chem. 9, 229 (1975).

${ }^{14}$ S. F. Boys and F. Bernardi, Mol. Phys. 19, 553 (1970).

${ }^{15}$ This conclusion might be altered if $R$ had been optimized at the SCF rather than MP2 level. It is this fact which is responsible for the slight irregularities in the SCF/6-31G** $(1 p, 2 d)$ values of $\Delta E$ along the conversion path. Analogous considerations apply to the MP2/4-31G* energies since $R$ was optimized at the SCF level for the smaller basis set.

${ }^{16}$ Previous electric deflection work had also detected a nonzero moment for $\left(\mathrm{NH}_{3}\right)_{2}$ : J. A. Odutola, T. R. Dyke, B. J. Howard, and J. S. Muenter, J. Chem. Phys. 70, 4884 (1979).

${ }^{17}$ A. D. Buckingham and J. A. Pople, Trans. Faraday Soc. 51, 1173 (1955); G. A. Bottomley and T. H. Spurling, Aust. J. Chem. 19, 1331 (1966).

${ }^{18}$ M. J. Frisch, J. A. Pople, and J. E. Del Bene, J. Phys. Chem. 89, 3664 (1985). 